\title{
The Order of Santo Stefano in the Levant: An Unpublished Account of a Voyage in 1627
}

Ilona Klein

Brigham Young University - Provo, ilona_klein@byu.edu

Christopher Kleinhenz

Follow this and additional works at: https://scholarsarchive.byu.edu/facpub

Part of the European Languages and Societies Commons, and the Italian Language and Literature Commons

\section{Original Publication Citation}

"The Order of Santo Stefano in the Levant: An Unpublished Account of a Voyage in 1627" (with Christopher Kleinhenz), Viator 21 (1990), 323-347.

\section{BYU ScholarsArchive Citation}

Klein, Ilona and Kleinhenz, Christopher, "The Order of Santo Stefano in the Levant: An Unpublished Account of a Voyage in 1627" (1990). Faculty Publications. 3822.

https://scholarsarchive.byu.edu/facpub/3822

This Peer-Reviewed Article is brought to you for free and open access by BYU ScholarsArchive. It has been accepted for inclusion in Faculty Publications by an authorized administrator of BYU ScholarsArchive. For more information, please contact ellen_amatangelo@byu.edu. 


\title{
THE ORDER OF SANTO STEFANO IN THE LEVANT: AN UNPUBLISHED ACCOUNT OF A VOYAGE IN 1627
}

\author{
by Ilona Klein and Christopher Kleinhenz
}

On 12 April 1627 a fleet of six galleys belonging to the Order of Santo Stefano set sail from Livorno on their expedition "contro il comune nemico"-the Turks. This journey would take them along the western and southern coast of Italy, through the Ionian Sea to the Peloponnese and across the Aegean Sea to the Dardanelles. Along the way the sailors encountered many vessels, both friend and foe, heard reports of sea battles and movements of ships in those waters, and listened to an account of a miracle wrought by the Virgin Mary. After having engaged in two fierce skirmishes with Turkish vessels, the fleet of the Order returned victorious to Italy, arriving in Taranto on 30 June 1627.

The history of the Order of Santo Stefano and of its various maritime activities in the eastern Mediterranean has been the subject of numerous scholarly investigations. ${ }^{1}$ Archival records for these activities abound, and many are available in published sources. ${ }^{2}$ However, in all of the published accounts that we have consulted, very little information has come to light about the voyage undertaken by the six galleys of the Order of Santo Stefano under the command of Admiral Giulio Barbolani de' Conti di Montauto in the period April-June 1627.

The fascinating and virtually complete account of the expedition is contained in a previously unpublished manuscript held in the Department of Rare Books and Special Collections in Memorial Library at the University of Wisconsin-Madison. By providing an edition of this text with accompanying English translation and

In the course of our research on this manuscript, we have incurred many debts, which we gratefully acknowledge here. We would like to thank John Tedeschi and his staff in the Department of Rare Books and Special Collections in Memorial Library (University of Wisconsin-Madison) for their endless technical assistance and moral support; Onno Brouwer, the director of the cartography laboratory, and his student assistant Dawn Burczyk for their generous financial and technical support in the creation of the accompanying maps; and Robert Karrow of the Newberry Library for allowing us to examine their wealth of cartographic materials. We give special thanks to the following friends and colleagues who shared with us their knowledge on a variety of matters pertinent to this project: Sarah Atis, John W. Barker, John Bennet, Diskin Clay, Kemal Karpat, Michael Petrovich, Louis Rossi, Domenico Sella, and David Woodward.

${ }^{1}$ Among the many works are the following: Roger Charles Anderson, Naval Wars in the Levant, 1559-1853 (Princeton 1952), and Gino Guarnieri, I Cavalieri di Santo Stefano nella storia della marina italiana (1562-1859), ed. 3 (Pisa 1960).

${ }^{2}$ See, in particular, the appendices in Guarnieri $289-482$. 
commentary, we hope to clarify many of the details concerning this important voyage. The route followed by the fleet and most of the place-names mentioned in the text are indicated on the two maps that accompany this article, one of the Italian peninsula and the other of the general Aegean area.

\section{THE Voyage}

Gino Guarnieri bases his history of the activities of the Order of Santo Stefano on numerous documents ("relazioni," "avvisi," "lettere," "resoconti," "patenti," "ordini," "memorie," etc.) held in the archives of Florence, Pisa, Venice, Genoa, and other cities. However, for this particular voyage Guarnieri apparently had at his disposal only a very few sources (and these from the Archivio di Stato di Pisa), which could not provide him with a complete picture of the events. Yet this voyage was, in his view, one of the most audacious maritime expeditions in the seventeenth century, particularly because of the presumed entry of the six galleys of the Order of Santo Stefano into the Sea of Marmara and their arrival before the city of Constantinople. ${ }^{3}$ This claim is based on a document ("Filza seconda di Convento et Navigatione dal 1624 al 1641") contained in the Archivio di Stato of Pisa which reads:

Sei galere pred.e sotto il med.o comando à 3 di giugno fecero di notte inuestire a... ... [sic] in Leuante un brig.o di turchi di Costantinopoli e fecero 11 schiaui.

L'istesse sei galere il med.o viaggio a 6 di giugno d.o 1627 presero alla Bocca e dentro il Canale di Costantinopoli 4 galeoni della Sultana, una Naue, et una germa

${ }^{3}$ Guarnieri (ibid. 198) expresses his view of the "importance" of this voyage in the following terms: "L'audacia e la fortuna incalzano, infondono animo e additano nuove mète da colpire: ecco la volta di Costantinopoli (Istanbul), ecco la più ardita, possiamo dire, delle audacie stefaniane.

"Anche questa volta, forzati i Dardanelli, penetrata nel Mar di Marmara e giunta all'ingresso del Bosforo, la squadra dell'ammiraglio Barbolani, composta soltanto di sei unità, all'altezza del Capo Giannizzero piombò addosso alla Carovana d'Alessandria di 22 vascelli, protetta da navi da guerra della "Sultana" e da un codazzo di altri legni da guerra minori. Eppure, i nostri Cavalieri, dopo non lungo contrasto, riuscirono a vincere la resistenza nemica, a catturare alcuni galeoni ed altri legni che rimorchiarono fin oltre lo stretto dei Dardanelli, nel Mare Egeo. E qui, se non fosse stata una violenta tempesta e l'inaspettato arrivo di una poderosa squadra turca di 16 galere mossa loro espressamente incontro da Rodi, la preda non sarebbe sfuggita di mano al Barbolani; ma per motivi di forza maggiore la si dovette abbandonare onde evitare un disastro irreparabile. Si dovettero sbarcare dai legni catturati tutti i contingenti stefaniani postivi a presidio, e si dovettero imbarcare di nuovo sulle nostre galere. Il che non si poté effettuare se non con manovra difficilissima e doppiamente pericolosa per il mare grosso e per le violente raffiche di moschetteria e di cannone del nemico. Ad ogni modo, non un uomo dei nostri andò perduto; la squadra dell'Ammiraglio Stefaniano tenne fronte valorosamente all'avversario e poté alfine disimpegnarsi con onore. Ma intanto rimane il fatto di questo nuovo audacissimo colpo nelle acque di Costantinopoli, e della gloriosa resistenza dei Cavalieri di Santo Stefano contro un nemico molte volte superiore in efficienza bellica. Nessuna potenza marittima mediterranea, per quanto grande, non soltanto ai tempi del Barbolani, ma neppure in tutto il secolo XVII, ardì penetrare nel Mar di Marmara e di presentarsi dinanzi alla Capitale dell'Impero Ottomano." These claims appear to be exaggerated, given the evidence provided by our document. 
moresca con altri sedici vascelli della Carouana d'Aless.a tra caramussali e saiche e fecero solo otto schiaui. ${ }^{4}$

(In the evening of 3 June the six above-mentioned galleys under the same command attacked... ?... [sic] in the Levant a Turkish brigantine from Constantinople and captured eleven prisoners. During the same journey, at the mouth and in the Channel of Constantinople, on 6 June 1627, the same six galleys took four galleons of the Sultana, a ship, a moorish germa with another sixteen vessels-including caramoussals and caiques-from the convoy of Alexandria, and captured only eight prisoners.)

Our document, which would appear to be an eyewitness account of the voyage, does not support Guarnieri's claims; indeed, it relates that the fleet of Santo Stefano did not proceed all the way to Constantinople, did not enter the Sea of Marmara, and did not even reach Gallipoli. On 6 June 1627, the fleet arrived at Capo Giannizzero, at the entrance to the Dardanelles, where it encountered and did battle with Turkish vessels, pursuing and capturing several of them in the channel. ${ }^{5}$ Other facts given in our "relazione" differ from those provided by Guarnieri's document. ${ }^{6}$ Consequently, we believe it necessary and appropriate to present them here in order to clarify the true circumstances of this 1627 sea journey.

Another reason for viewing Guarnieri's statement with suspicion is the account given by Roger C. Anderson who, not using Guarnieri's work as a source for his own research, relates that the

Tuscans, still commanded by Montauto, returned to the Levant in 1628. After landing in Asia Minor at the beginning of June, their 6 galleys took a number of small Turkish ships quite near the Dardanelles, but only to have to abandon them on being overtaken by a force of 16 Turkish galleys from Rhodes. ${ }^{7}$

These events bear a striking similarity to those recounted in our document; indeed, this account of the capture of Turkish vessels in or near the Dardanelles and their subsequent abandonment appears to present in abbreviated and telescoped form the historical facts. However, given the evidence of our text and the documents provided by Guarnieri, the date that Anderson gives - 1628 - is incorrect and should be 1627. Although skirmishes did occur in 1628, none matches Anderson's description. From the general nature of his account, it would seem that Anderson was working from vague and inaccurate secondary sources, rather than from reliable archival documents.

Taken together, the contradictory accounts of Guarnieri and Anderson lead us to

${ }^{4}$ Ibid. 364, doc. no. 43

${ }^{5}$ We believe that Guarnieri in his perhaps overly enthusiastic assessment of the accomplishments of the Order of Santo Stefano misinterpreted the phrase in his text- "alla Bocca e dentro il Canale di Costantinopoli" - to refer to the area around Constantinople. While the phrase "canale di Costantinopoli" can be used to designate the entire Sea of Marmara, it can also refer simply to the Dardanelles, as it does here in our text.

${ }^{6}$ For example, while Guarnieri reports the taking of eight prisoners in this battle, our text speaks only of seven.

${ }^{7}$ Anderson (n. 1 above) 113. 
believe that our text may be the only surviving, extended document of this voyage. The expedition of the Order of Santo Stefano to the Levant was probably well known because of the great booty taken (and subsequently lost). ${ }^{8}$ However, up until our recent identification of this manuscript, no reliable first-hand evidence for this voyage has been available. Although Guarnieri generally cites numerous archival documents to support his interpretations, for this particular voyage he refers to only the "Filza seconda ...." quoted above. Given his usual care in assembling and referring to archival documents, we are persuaded that there are no other extant sources relating to this expedition, at least among those listed and consulted by Guarnieri. The lack of documentation for this voyage may also be noted in Guarnieri's transcription of the "Registro delle Prede" which lists the major material conquests by the Order of Santo Stefano between 1568 and 1688 but does not mention any between February 1626 and June $1628 .^{9}$

\section{The MANUSCRIPT}

The manuscript (\#192 uncataloged) bears the title "Viaggio fatto in Levante dalle 6 Galere dell' Ill.ma Sacra Relig.ne di s.o Stefano del ser.mo Granduca di Toscana q.o Anno 1627 sotto la Carica dell Ill.mo Sig. Giulio Barbolani de' Conti di Montauto Gent. \&c." It came to Memorial Library as a gift of Mrs. Edmund K. Nielsen of Appleton, Wisconsin, in 1984. Her son, Edmund B. Nielsen, an art historian, acquired it from an unknown source in Florence in 1950.

The manuscript consists of a single sheet of paper, folded in half, forming four unnumbered pages measuring $20.5 \times 30.5 \mathrm{~cm}$. each. The sheet has a watermark in the shape of a tear drop containing the outline of a Latin cross. ${ }^{10}$ The text is written in light brown ink on the first three pages, in a very small regular cursive script contemporary with the events described. In addition to a relatively few corrections of individual letters and words, the text displays one obvious modification: the last two lines of the second page have been completely crossed out, rendering the original text illegible, and two lines in the same hand have been

\footnotetext{
${ }^{8}$ The narrator of our text notes the incredible size of the booty, exclaiming: "Presa veramente grande che a Memoria di homini no(n) è Mai stata fatta dalla armata intera, che p(er) quanto si intese dalli stiavi fatti, passava il Valore di piu di 700,000 scudi." (This was truly impressive booty: indeed, in human memory booty of this magnitude has never been taken by the entire fleet, and from what we were able to learn from our prisoners, the value surpassed 700,000 scudi.)

${ }^{9}$ Guarnieri (n. 1 above) 457-468, doc. no. 113. With regard to this important record, Guarnieri 17 notes its unfortunate loss, "distrutto dagli ultimi eventi bellici, preziosa fonte storica, ma poiché esso 'Registro' fu da me a più riprese copiato e pubblicato ed oggi riprodotto nell'appendice documentaria di questo volume, quasi nella sua integrità, così può dirsi non essere assente quella generosa fonte informativa."

${ }^{10}$ We have not been successful in identifying this watermark in either Charles Moïse Briquet, Les filigranes: Dictionnaire bistorique des marques du papier des leur apparition vers 1282 jusqu'en 1600, ed. 2 (Leipzig 1923), or in Edward Heawood, Watermarks Mainly of the 17th and 18th Centuries, Monumenta Chartae Papyraceae 1 (Hioversum: Paper Publications Society, 1950). The watermark is not easily decipherable; it may be surmounted by a crown, and may have cursive letters directly below the point of the tear drop. What appears to be a combination of capital letters may instead be an Agnus Dei or another variety of animal.
} 
substituted between the lines. The sheet is loosely sewn into a light blue cardboard binding of very recent date.

The records of voyages in this period vary, from short, concise lists of names and places to extended narratives of expeditions undertaken. Our document presents more than a simple, abbreviated summary of the itinerary and major events; indeed, it provides a fully developed story which conveys to the modern reader the numerous hardships, dangers, and adventures of sea voyages in the early modern period. Moreover, it offers what appears to be an eyewitness account of several naval battles, as well as a secondhand account of a miracle which took place in Constantinople. There are, in this text, elements of potential interest to readers in many fields: for example, to historians, for the political composition of the area, martial activities, number and types of vessels employed in the Mediterranean, commodities traded, and daily activities of the crew; to geographers, for the placenames and navigation routes; to linguists, for information on seventeenth-century syntax, grammar, and vocabulary, including specific nautical terms and loan words; to students of literature, for the various narrative techniques employed in the text; and to hagiographers, for the account of the miracle of the Virgin Mary.

Throughout his narration of the vicissitudes of the sea journey to the Levant, the author/scribe remains anonymous. The narrator tells the story using the impersonal si. Only once does he enter the narrative in the first person singular, when he justifies his detailed description of a miracle which occurred at Constantinople. However, he often uses the collective first person possessive plural pronoun (nostri, nostra, etc.). In our translation we have consistently used the first person plural pronoun "we" instead of the impersonal form, in order to facilitate comprehension of the events.

From a linguistic point of view, the text, written in a Tuscan dialect, has features characteristic of the period: 1) retention of etymological $b$ (bavere, buomo) with the sporadic and unnecessary appearance of $b$ elsewhere (barcha, chredeva); 2) lack of substitution of $z i$ for $t i$ (pretiose, spatio); 3) some oscillation between single and double consonants (fugirelfuggire, arivolarrivo) with a distinct tendency toward simplification (quatro, vechio) and very few geminates (doppo, robbe); 4) retention of older third person plural preterite forms in -orno (tornorno, confermorno) and -eno (disseno, viddeno, feceno), and simplified $-m m$ - in the first person plural (andamo); 5) retention of older third person plural imperfect subjunctive forms in -sseno (bavesseno, fusseno). Other peculiarities include: 1) rhotacism of preconsonantal $l$ (sarpare); 2) metathesis of postconsonantal $l$ (replubica) and $r$ (riscronto, scropendo); 3 ) the typical development in Tuscan of initial sk i to st $i$ (stiavi); and 4) the common Tuscan use of the first person plural imperfect eramo (<eramus).

In tracing the sea route of the expedition, we encountered some minor difficulties with place-names. On certain occasions the Greek names of harbors and islands appear in their Italianized form. At times the toponym is a direct translation: for example, Isola dei Cervi from Elaphonisos ( $<$ Gr. elaphos = deer, stag); at other times the name is a phonetic transposition from Greek to Italian: for example, Zia for Kea, and Scopullo for Skopelos. On at least one occasion, the place-name reflects a distinctive topographical feature: Capo Colonna (= Cape Sunion) takes its name from the columns of the Greek temple of Poseidon, which are still visible from the sea. Foreign words indicating military titles and types of ships are 
transliterated phonetically into Italian: for example, chîâa from the Turkish kâbya and germa from Arabic garm.

In the wake of recent interest in "travel literature," we believe that the lively narrative style used by the scribe and the exciting maritime adventures described will make this text appeal not only to Italianists, but also to a broader readership. The critical edition contains the transcribed seventeenth-century manuscript in its entirety, together with an English translation. While the notes to the Italian text treat paleographical matters, those to the English translation explain matters of interpretation (geography, maritime terminology, etc.)

In establishing the present edition, we have maintained the distinction in the manuscript between upper- and lower-case letters, expanded all abbreviations (within parentheses), distinguished between $u$ and $v$, and added punctuation and diacritical marks according to modern usage. In the English translation we have attempted to be as literal as possible; yet, in the interest of comprehension, we have divided the lengthy and often difficult Italian periods into more manageable sentences.

We hope that this document "Viaggio fatto in Levante. . . nel 1627" may enrich the growing collection of early travel literature which is currently being rediscovered in European and American archives.

\author{
Department of Foreign Languages and Literatures \\ Loyola College in Maryland$$
\text { Baltimore, Maryland 21210, U.S.A. }
$$ \\ Department of French and Italian \\ University of Wisconsin-Madison \\ Madison, Wisconsin 53706, U.S.A.
}

\begin{abstract}
TEXT
Viaggio fatto in Levante dalle 6 Galere dell'Ill(ustrissi)ma e Sacra Reli$\mathrm{g}$ (io)ne di s(ant)o Stefano del ser(enissi)mo Granduca di Toscana q(uest)o Anno 1627, sotto la Carica dell'Ill(ustrissi)mo Sig(nor) Giulio Barbolani de' Conti di Montauto Gent(iluomo) \&c.
\end{abstract}

[1] Doppo havere il dì 12 di Aprile spalmato in Livorno, et essersi allestito di quanto era necessario p(er) fare secondo l'uso Loro, le 6 Galere, p(er) il corso contro il comune nemico, no(n) poterno $\mathrm{p}(\mathrm{er})$ il sinistro tempo fino a 19 partire, come fecero, di conserva della Galera S(ant)a Maria dell'Ill(ustrissi)ma e sacra Relig(io)ne di Malta, e della nuova Galera s(ant)o Carlo, donata alla predetta Relig(io)ne dal ser(enissi)mo Principe Don Gio(va)n Carlo Medici gran Priore di Pisa.

[2] E la sera medesima alle 3 hore di notte, si giunse in Portoferraro, lasciando quivi il novello S(ign)o(r) Principe di Piombino e li Pagatori. Ma p(er) il Vento contrario no(n) si pottette partire fino alla Matt(in)a de 20 alle 10 hore, facendo vela alla volta del monte Argentario.

[3] E preso la spiaggia romana, fu forza $\mathrm{p}(\mathrm{er})$ il sinistro tempo entrare la 
Matt(in)a de 21 in Civita Vechia; onde no(n) si potette partire fino a 26 alla diana, che, passato la spiaggia romana felicem(en)te, ritornando il Vento e Mare contrario, bisogniò dar fondo a Gaeta, et ivi si stette fino alla Matt(in)a de 28, che fatto vela. [4] Quando si arivò a Nisita, si lasciorno le Galere di Malta, che, doppo salutato La Capitana, andorno verso Napuli, e noi, tirando a Camino, andamo a Capri, che mettendosi mare e Vento contrario si diede fondo. Ma pegiorando tuttavia, a 30 si tornò adietro a dar fondo al Mar Morto, donde no(n) si potette partire fino al dì 4 di maggio avanti giorno.

[5] E traversando il Golfo di salerno, si entrò la Matt(in)a de 5 in Messina, et il giorno seguente su la sera si fece Vela tirando p(er) Levante, trovando a 7 a l'alba una Pollacha fransese che veniva di smirne, et era 12 giorni che mancava, et andava a Marsilia. Diede nuova che il Bei di Rodi era passato a Costantinopuli con 14 Galere, et che anco la Carovana di Alesand(ri)a non era partita, e che p(er) tutto levante era buona sanità.

[6] Et lassato a suo viaggio, si seguitò a Camino. E nel Golfo di squillacie si scoperseno allo Mare 3 Vascelli quadri, ma p(er) esser il Vento fresco et il mare grosso e l'hora tarda non si riconobero. E si andò la sera a dar fondo a Cavo Colonna, e la Matt(in)a delli 8 di nuovo si scoperse 3 Vascelli, che si giudicorno li stessi. E sarpando $\mathrm{p}(\mathrm{er})$ andare a riconoscerli, ${ }^{a}$ si tornò al Cavo $\mathrm{p}(\mathrm{er})$ esser il temp(o) cattivo. E dimorando quivi fino a 9 alla diana, si fece Vela, et l'istessa sera si entrò in Taranto. E dato ordine di spalmare, si differì alli 12 per il mal tempo. Et spalmato il dì 3 Galere, il dì 13 si spalmorno l'altre 3.

[7] Et rifatto le stive di Biscotti, Vini, Carne et altri rifreschi, alla prima di notte si sarpò verso C(apo) s(ant)a Maria, ma mettendosi mare grosso e vento freschissimo contrario, fu forza dar fondo a Galepoli a 15. E dimorato quivi tutto il resto del giorno e parte della notte, si sarpò, e a 16 si diede fondo a C(apo) s(ant)a Maria. Ma mettendosi la notte Mare e Vento sinistro con fortuna improvisa, p(er) fuggire la traversia fu necessario su la mezzanotte sarpare e mettersi allo Mare, e tornare a Galipoli, quale si afferrò La Matt(in)a de 17. E si diede fondo a l'Isola, et mettendosi il tempo allo stesso segno con gran malignità, trattenendosi quando a l'Isola e quando alla Città.

[8] Ritornando il temp(o) a Bonaccia, a 20 si sarpò, e la matt(in)a de 21 si surse al C(ap)o sudetto, e circa alle 17 hore, venuta la guard(i)a mandata in terra, e condusse alla Cap(ita)na un huomo del paese, che diede nuova al s(ignor)e Generale come era stato spedito dal Governatore di Otranto l'istessa mattina p(er) avisar la Costa, che stesse con buone guard(i)e, stante l'essersi scoperto l'istesso dì allo Mare 13 Galere, che venivano del Golfo alla volta del Cavo, che si stimavano fusseno Barbaresche. Et il sig(nor) Generale ne fece avisare le Galere, che universalm(en)te si sentì tal nuova vana, e, se pure era vera, fussero Galere Venetiane.

[9] E dimorati in quel Lu[o]go fino alla 3 di giorno, si scoperse fuori del Cavo un Vascello quadro, quale messo in Caccia, et esso, scoperto le Galere, armato la Barcha, abandonandolo, si salvorno le persone in terra, ma-assicurati-tornorno a Bordo: era una Nave Venetiana, et era quatro giorni che partiva di Manfredonia carica di grano p(er) Napuli. Né diede nuova alcuna.

[10] E lasciato a suo Viaggio, et essendo il tempo buono, con il consiglio di 


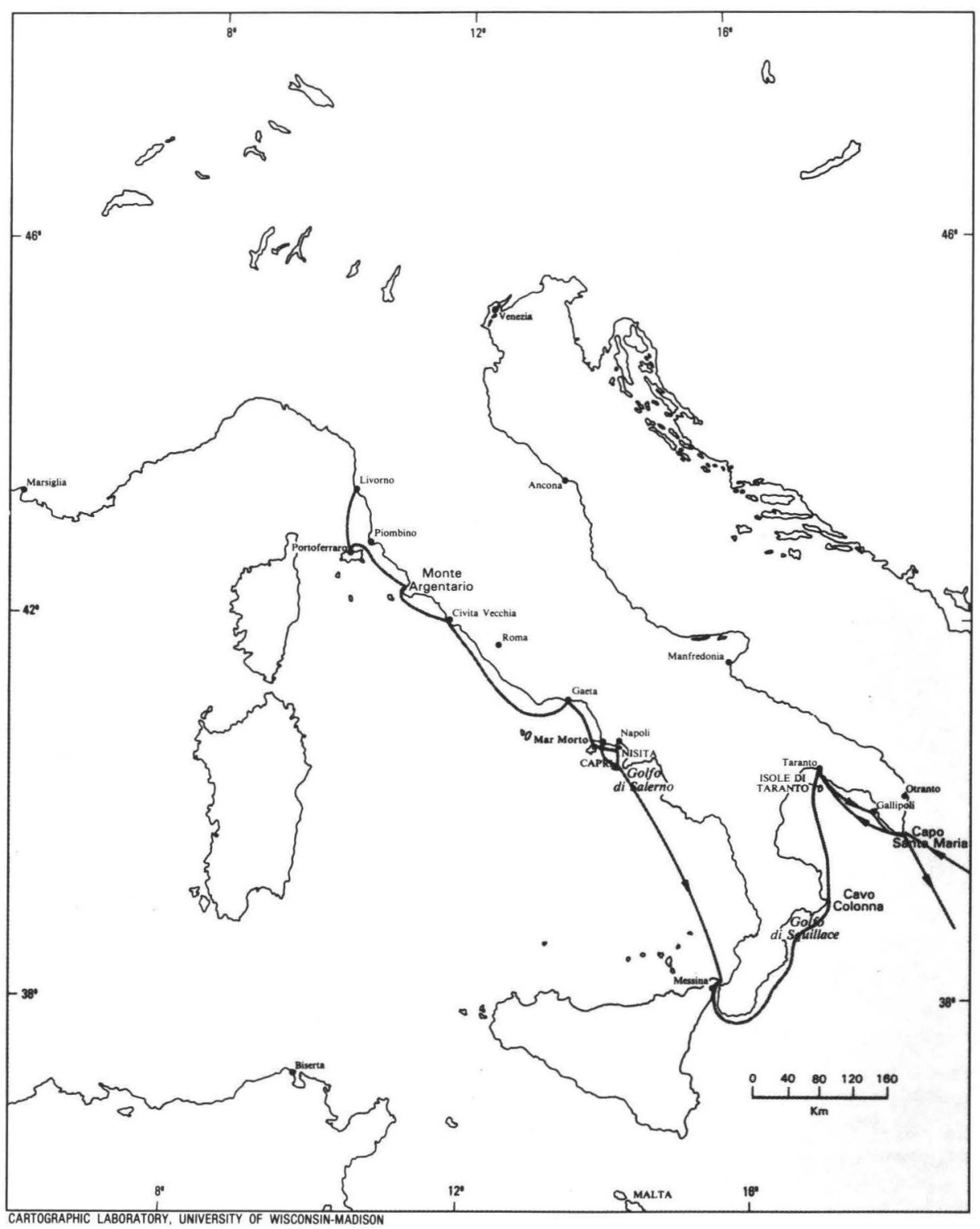

MaP 1. The Italian Peninsula

tutte $^{b}$ le Galere si fece la parenzana, et traversando il Golfo travagl(iat)o un poco $\mathrm{p}(\mathrm{er})$ il mare la notte, a l'Alba de 22 si afferrò la Ciaffalonia, e, piglato u(n) poco di reposo, la stessa sera alle 23 hore, si arrivò al Zante. E surti alla spiaggia e Mandato la Filuca a Piglare lingua alla Città, tornò e referì come dui mesi avanti, in spatio di 13 giorni, era stato ribellato da Crestiani stiavi due Galere Turchesche, cheforzate poi dal temporale - erano corse in Cand(i)a. Di una delle quali, che era del 


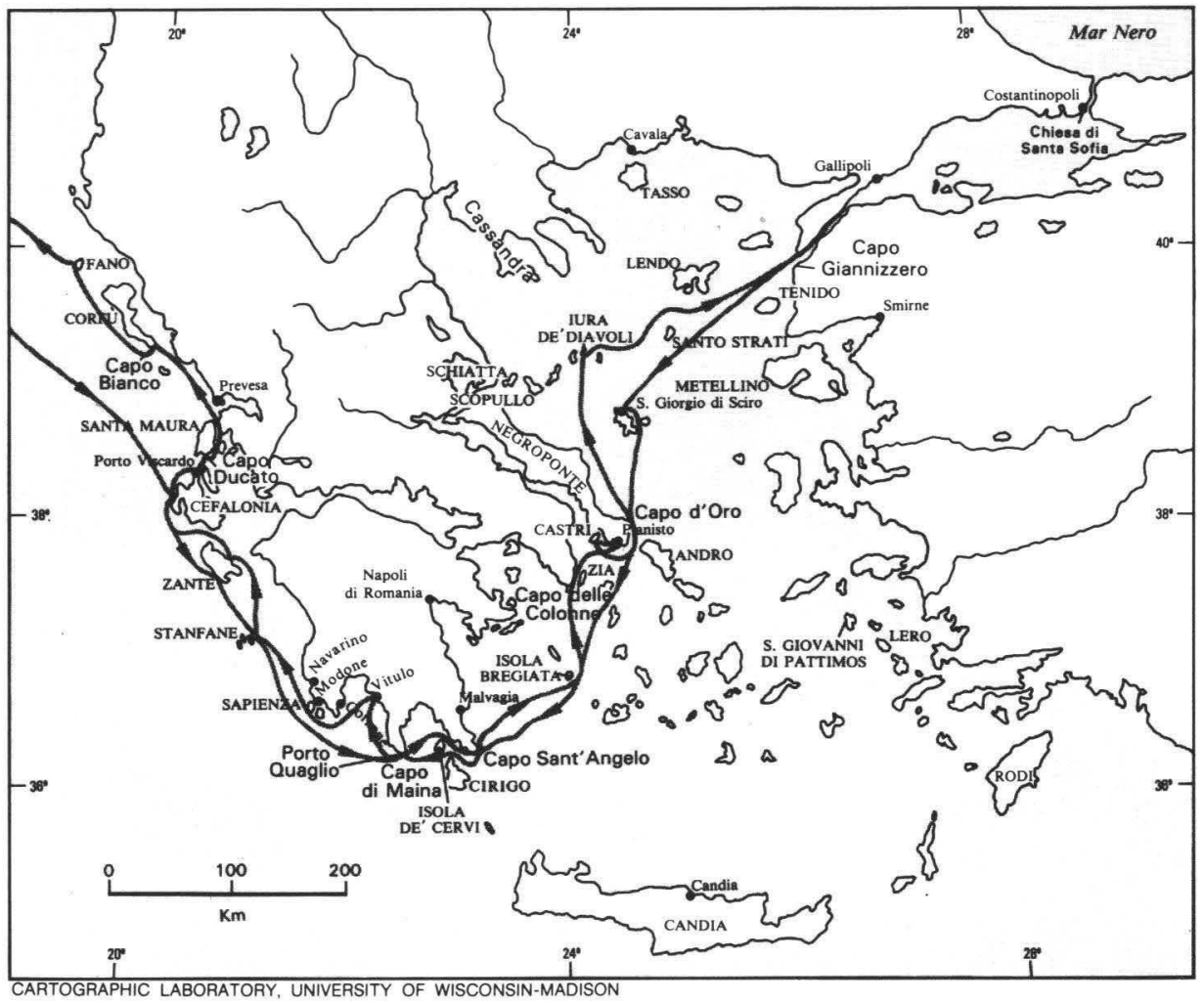

MAP 2. The Ionian Sea to the Dardanelles

Bei della Cavalla, s'erano salvati tutti i Cristiani, ' dua de' quali il s(ign)o(r) Generale imbarcò sop(r)a le Galere p(er) esser casato in Livorno; e l'altra Galera, che era di Negro Ponte, era stata resa al Turco.

[11] E dato parte alla notte, a 23 a giorno si diede fondo alle stanfane, e Celebrato la s(ant)a Messa, fatto rifresco de l'Acqua, doppo desinare si fece Vela [a] C(apo) di Maina, dove dato fondo alli 24. Si entese ${ }^{d}$ da' Mainotti che a Corrone era una Galeotta di Turchi di 24 Banchi e u(n) Bregantino di 16 stando. Surti quivi, e venendo dui altri Mainotti, che dissero che al C(ap)o s(ant)o Angelo era 3 scaiche et $\mathrm{u}(\mathrm{n})$ Caramussale, che chredeva fusseno Turchi e quivi havesseno dato fondo.

[12] Onde p(er) ciò il s(ign)o(r) Generale mandati a riconoscerlj p(er) la Filuca, quale tornò e referì esser vero che quivi erano sul ferro; onde fatti armare 4 Caechi, li mandò insieme con la Filuca a quella volta, acciò no(n) si salvasseno se fusseno stati Turchi in Terra, e sarpò detro loro con le Galere.

[13] Ma venuta l'alba, vennero le Barche di essi alle Galere, e disseno che erano Vascelli di Greci di s(ant)o Gio(vanni) di Pattinos e di Mitellino. No(n) havevano turchi né meno robbe di Loro, sì come si giustificò: havevano Cotoni filati e Vallonie, che havevano carico a Lero per Ancona e Venet(i)a, erano 12 giorni che 
mancavano. Diede nuova che ne l'Acepelago no(n) erano aloro che 7 Galere con il Bei di Rodi, essendo l'altre passate al mar nero con c(irc)a 4 Vascelli piccoli p(er) la nuova mossa di Arme fatta in quelle parte da' Rossi; e che d(ett)o Bei haveva accompagnato u(n) Galeone carico di Polvere, Palle di Cannone et altre Munitioni da Guerra quasi fino ad Alesandria p(er) passare al Campo Turchescho in Asia, dove si sentiva no(n) solo il Persiano a' danni del Turcho, ma molti ribelli; che p(er) tutto era buona sanità.

[14] E Lasciati a Loro affari, si diede quivi fondo, ma poco doppo la Guard(i)a di terra fece segno di Vascelli Latini e, venuta alla Capitana, referì che fuori de l'Isola de' Cervi erano quatro Vascelli Latini, che dalla grand(ezz)a e Camm(ino) grande si stimavano che fusseno Galere; onde si sarpò a quella volta. Fuori del cavo S(ant)o Angelo si scoperseno dui Vascelli quadri che, messi in Caccia e riconosciuti, erano 2 Caramussali di s(ant)o Gio[vanni] di Pattinos, che venivano da Zia, erano 5 giorni che mancavano, et havevano Vallonia p(er) Venetia. E disseno che sul mezzodì havevano trovato in Canale tra il Cirigo e l'Isola de' Cervi 2 Galeazze Venetiane che andavano al Cirigo.

[15] Lasciati al Loro Viaggio, si seguitò a camino, e la $a^{e} \operatorname{matt}($ in)a de 26 si afferrò l'Isola Bregiata; e riposato le Curme, su le 20 hore disarborando, si tirò verso il Cavo delle Colonne. E datovi fondo la mattina del 27 vi si trovarno 2 barchette di Greci pescatori di Malvag(i)a, di donde mancavano 3 giorni. E disseno che di Napuli di Romania 6 Galere di più Bei là havevano spalmato, et erano passate a Costantinopuli p(er) andare al mar nero a' danni de' Rossi, che con gran numero di scaiche facevano molti progressi nelli paesi turcheschi, e che del medesimo Luogo erano 6 giorni che s'erano partiti 3 Caramussali con 600 Giannezzeri p(er) lo stesso effetto.

[16] Et Lasciati quivi, si fece vela la sera verso di Negro Ponte, et alla diana il dì 28 si ar[i] vò a Planisto. E fatto acqua reale alla fiumara, si turnò adietro p(er) il mal tempo a dar fondo a Castri. Nella stessa Isola, dove si trovò un Caicho, carico di formaggio e olio di Turchi e Greci [ . . . . . $f$ Et i Turchi si salvorno in terra. Si prese quello che era di Turchi, e si lassò l'altro; né si intese nuova di momento, se no(n) che veniva di Napuli di Romania.

[17] E Mentre si stava sul ferro, sul mezzodì arivò quivi uno stiavo Christiano greco, solo, con una Barchetta, fugito di Zaychara, et era 4 giorni che ne mancava; e da ess[o]s si intesero le stesse nuove che $\operatorname{sop}(\mathrm{r}) \mathrm{a}$, et il s(ign)o(r) Generale, imbarcatolo sop(r)a La Capitana, fece sfondare la Barchetta.

[18] E stando quivi il dì 31, si scoprì in Canale p(er) la parte di Andro un Vascello Latino, ma il Vento freschissimo et il mare grosso no(n) permisseno l'andare a riconoscerlo. E finalmente abonaciato il mare e Vento, il dì 2 giugnio si sarpò doppo desinare verso C(apo) d'Oro, e datovi fondo a riposare le Curme, alla prima di notte si sarpò verso l'Isola di Gazzela, che il dì 3 a l'alba si afferrò, dove, dimorando, la guardia di terra venne a dar nuova di u(n) Vascello [page 2] adosso del quale_- p(er) esser piccolo_-si fece uscire la filuca con u(n) Caecho armato, che lo presero e rimburciorno alla Cap(ita)na. Era di Greci del Tasso, e da loro si intese

'MS la written over li

There appears to be a lacuna at this point in the text.

${ }^{8} \mathrm{MS}$ esse 
era 10 giorni che mancavano, et andavano a scopulo a Vender Pece, e confirmorno le stesse nuove che $\operatorname{sop}(\mathrm{r}) \mathrm{a}$.

[19] E lasciati in quel luogo con ordine di no(n) ripartire di quivi, l'istess[a] ${ }^{b}$ sera si fece Vela verso Iura de' diavoli, ove arivati su la Mezzanotte, con la Filu(c)a che andava a riconoscere la Cala, la quale scoperse u(n) Caecho Armato alla stessa Isola; che, vedute le Galere, appresso andò ad investire in terra, buttandosi allo Mare p(er) fretta 3 Turchi che furno dalla medesima Filuca ricuperati ${ }^{i}$ e condotti alla Capitana, salvandosi altri 13 al terreno, restando ivi dentro l'Agà di s(ant)a Maura, che, p(er) esser Vechio e zoppo, no(n) potette fugire. Ma volendo di nuovo tornare la Filuca a Bordo al Vascello, il d(ett)o Agà prese u(n) Moschetto carico con Palle e Palloni grossi e lo sparò a' nostri, ferendo quanti in essa si trovavano de' $\mathrm{n}$ (ost)ri e quasi tutti Mortalm(en)te, uno de' quali morse di quivi a pochi giorni che fu il Patrone della Filuga. Gli altri al n(umer)o di 13 Turchi, superate le dificiliss(i)me salite p(er) quelli aspri scogli, si salvorno alla Montagna con il Benefitio della Notte. E venuto il giorno, si Misseno in terra soldati e Marinari, che in poche hore condusseno altri 6 turchi alle Galere, no(n) essendo stato possibile trovare gli altri. E sop(r)agiunto la notte, si fece ritirare tutta la gente alle Galere, restando uno scocho in terra de' nostri che si chredé o fusse Morto da' Turchi fugiti, o che si rompesse il Collo in qualche precipitio.

[20] Preso lingua, si intese era $u(n)$ Caeccho che veniva di Galipoli e la Matt(in)a avanti era partito, che sop(r)a haveva 17 passegiri Turchi, e fra essi il sop(r)anominato Agà et il chîaîa del Capita(n) Bascià che no(n) fu trovato; che erano $^{j} 15$ giorni che erano ${ }^{k}$ passate al Mar nero 10 Galere con 40 fregate, et che il Generaliss(i)mo Bascià con altre 50 Galere era di partenza anche esso p(er) lo stesso effetto, aspettandosi che il dì 4 di Giugnio di Venardì uscisse a quella volta. Che era passato contro il persiano Alì Bascià con 150,000 Turchi, e che p(er) tutto era buona sanità. E, svaligiato il Vascello, scropendo 2 altri Vascelletti di Greci carechi di grano, che havevano carecato a Cassand(r)a, e andava a Napuli e Malvagia. E Venuto la notte, si fece Vela verso il Tenido, sarpando la Mattina de 5. Sul far del giorno, due scache in Canale tra Lendo e s(ant)o strati, quali messo in Caccia e so[p]ragiunti, riconosciuti erano di Gre[c]i di schiatta e veniva su la matt(in)a da s(ant)o stratti, e cinque giorni erano che mancavano di Costantinopuli. Disseno che la Matt(in)a avanti a d(ett)a Isola havevano trovato una Galeotta, quale, doppo di haverla ${ }^{l}$ presa rifrescho, era andata $\mathrm{p}(\mathrm{er})$ entrare dentro in Canale alle bocche del quale si trovavano c(irc)a 34 Vascelli tra Turchi e Christiani; parte di quelli erano della Carov(an)a di Alesand(ri)a, et che fra essi erano 2 Galeoni e quatro Urche che no(n) potevano $\mathrm{p}(\mathrm{er})$ li Grecali freschi entrare. E confermorno le stesse nuove della armata turchesca e dello esercito contro il persiano come sop(r)a [ . . . ]. ${ }^{f}$

[21] Affermando alcuni Caloiri con giuramento loro come nella Chiesa di s(ant)a soffia - oggi profanata in Mos[c] hea ${ }^{m}$ di Turchi-s'era miracolosam(en)te

\footnotetext{
${ }^{b} \mathrm{MS}$ 1.istesse

${ }^{i} \mathrm{MS}$ riconosciuti, crossed out

iMS herano

${ }^{k} M S$ herano

'MS haverla written over havere

${ }^{m} M S$ Moshea written over Moshett
} 
scoperto una immagine della Gloriosa ${ }^{n}$ Mad(r)e Vergine Maria, dipinta in Tavola, che, da' Turchi veduta, fu buttata in Mare. Ma retornata al medesimo Luogo con Miracolo stupendo e da' Turchi veduta, e tra gli altri u(n) Chiausse, facendola di nuovo Levare considerata, domandò che cosa fusse quella a u(n) Giannizzero; fattosi avanti p(er) divina voluntà, disse quella essere una immagine della Vergine Maria Madre di Christo dio de' Crestiani. Onde il d(ett)o Chiausse, cheamandolo "Cane!" messe mano alla scimitarra, uccise il Giannizero. Spezzato l'immagine sacra in più pezzi insieme con il $\mathrm{d}(\mathrm{ett}) \mathrm{o}$ Morto, lo fece buttare in Mare. Ma la Matt(in)a seguente, di nuovo entrati i turchi nella Moschea p(er) fare le Loro erronee ciremonie, viddeno il Giannizzero Ginochioni avanti alla immagine intera, ritornata al suo Luogo sana come prima e, di più, sop(r)a essa una Croce. Onde fatto subito di ordine del gran signore serrare la chiesa, impossero pena a chi, sì turco come christiano, s'acostasse o entrasse dentro, dando bere o Mangiare da uno angusto Luogo a d(etto) Giannizze(r)o, che solo vi asisteva con havere fatto fare il gra(n) sig(nor)e una lampada d'Oro che del continouo arde. Ho voluto descrivere questo Caso, acciò con più chiarezza possa intendersi la pura Verità del commesso miracolo occorso, di che si fanno come di gran prodigij in Costantinopuli gran bisbiglio.

[22] E Lassati a Loro Viaggio, si seguitò a Camino, così navigando tutto il giorno, tutta la notte seguente. Essendo stati disarborati tutto il giorno, la matt(in)a de 6 a giorno si giunse a Giannizzero, \& scoperto i nominati Vasselli, 22 di quelli erano di Turchi fra Urcche, Caramussali, Germe, e Scaiche. Vedute le Galere, furno abandonati da Turchi che si salvorno in terra.

[23] E lasciata la Pat(ron)a, S(ant)o fran(ces)co, e s(ant)a Maria Mad(dalen)a alla Maggiore parte di essi, che erano alle boche, con altre 3 Galere si imbocò dentro, seguendo la Caccia di altri che cercavano la salvezza. Ma ancora essi furno abandonati, che era u(n)'Urcha con 7 Caramussali; quali Vascelli, cavati i Migloramenti, insieme con la Urca, si rimburciorno fuori ${ }^{0}$ e $\mathrm{si}^{p}$ dette fondo al pred(ett)o Cavo. E levati i migloramenti che erano se bene pochi delli Caramussali e saiche, il s(ign)o(r) Generale fece Marinare 4 Urche, una Germa grossa, una Pollacha, careche tutte di riche Merci e pretiose Cose, havendo fatto solo 7 stiavi, et abandonando gli altri alla Ventura del Mare, che altro no(n) havevano che risi e cose simili.

[24] Il seguente giorno si sarpò su la sera, rimburciando ciascheduno il suo. Presa veramente grande che a Memoria di homini no(n) è Mai stata fatta dalla armata intera, che $\mathrm{p}(\mathrm{er})$ quanto si intese dalli stiavi fatti, passava il Valore di più di 700,000 scudi.

[25] E tirando avanti, la sera delli 9 il Cattivo tempo ne sforzò a piglare porto a s(ant)o Giorg(io) di sciro. E la matt(in)a del $q$ 10, mancando l'acqua, si andò ad altra Cala poco lontana, e si fece. Lassato quivi i Vascelli, e ritornando alla posta, si scoperseno due scaeche allo Mare che venivano a pigliare terreno; quale avicinate e riconosciute, erano di Greci di Molivo de l'Isola di Mitellino, cariche di Vallonia 
p(er) Venetia. Diedero nuova come, essendo Loro la domenica passata a d(ett)o luogo, arivò quivi un Caecho armato spedito con diligenza da l'Agà del Tenido ad avisare il restante della Carovana che era adietro-cioè u(n) Galeone con altri Vascelli e 3 Galere careche di gra(n) tesoro-che p(er) aviso rimburceando sotto le $\mathrm{d}(\mathrm{ett}) \mathrm{e}$ Galere il pred(ett)o Galeone. E gli altri Vascelli quivi havevano dato fondo, asserendo che havevano sentito di quel Luogo il rumore delle Cannonate. Né diede altra nuova, e venuti anche essi a dar fondo.

[26] Ma tornando il tempo favorevole su la mezza notte, si uscì e si fece Vela verso $\mathrm{C}(\mathrm{apo})$ d'oro, che paregiato si tirò avanti e, passato l'Isola Brugiata, a 13 a mezzogiorno fu necessario dar fondo a C(apo) s(ant)o Angelo, dove venne u(n) Albanese, e diede nuova come era 10 giorni che Aptì Bascià era con la sua Galera venuto di Barberia e, giunto a Modone, che stante le nuove che haveva che eramo dentro, s'era fermate. E su la sera venne quivi u(n) Caecho dal Cirego con gente del Luogo, e disse che sette giorni avanti vinagro con una galera era passata verso Barbaria. E su la Mezzanotte si sarpò, et si andò a l'Isola de' Cervi, e p(er) il sinistro tempo bisogniò dar fondo.

[27] E stando in quel Luogo, essendosi scoperto allo Mare fuori del Cirigo u(n) Vasello quadro che, uscite le Galere a riconoscerlo, era Inglese: veniva di Livorno et erano quatordici giorni che Mancava et andava a smirne, havendo fatto fino sop(r)a Navarino conserva con il Vascello delli 11 Cavaliri fra' Gualterotto Bardi e fra' Nicolò Gionfigliazzi, et che erano 3 giorni che s'erano spartiti.

[28] E Lassato al suo Viaggio, si tornò alla posta. E dato parte alla notte, si fece vela verso c(apo) di Maina dove si giunse la Matt(in)a delli 15. E dimorando quivi tutto il giorno e parte della notte, volendo uscir fuori, no(n) fu possibile $\mathrm{p}(\mathrm{er})$ il mal tempo et anco p(er)ché era imbarazato uno delli Vascelli con il ferro della Capitana, che stette c(irc)a 7 hore in quel travaglio.

[29] Ma volendo a l'Alba partire, la Matt(in)a de 16 si scoperse alla punta $\mathrm{p}$ (er) Levante una Galera che veniva alla volta del Porto, che subito il segnor Generale fece sarpare La Patrona, santo Fran(ces)co, S(an)to Stefano, essendo stato necessario, p(er) essersi di nuovo imbarazato la Gumina della Capitana, Lasciarla p(er) ochio. [30] Et havendo anco sarpato la S(ant)a Maria Mad(dalen)a e s(ant)a Crestina, e si uscì fuori, ma La Galera s(ant)o Fran(ces)co, come più fuori delle altre, scoperse altre Galere. Sparò u(n) tiro con far segno, sì come fece la Galera predetta alle Conserve, che subito cominciorno a parir fuori della punta: che furno altre 14 Galere di fanale che con gran Vigoria venivano alla volta nostra p(er) socquadrarne et investirne, tirando molte Cannonate, senza nostro danno come a dio Piacque. [31] Ma seguitando la Galera nemica alla volta della Cap(ita)na, con la quale trovò duro riscronto di freque(n)tiss(i)me Moschetate, et anco non la poteva arivare come si presupone farsi. Ricevendo danno e temendo di peggio, ${ }^{r}$ levò remi e salpò. ${ }^{s}$ Et uscendo La Cap(ita)na a vantaggio, la galera sud(ett)a si voltò alla s(ant)a Crestina; che visto dal s(ign)o(r) [page 3] Generale la Caccia tanto stretta, fece segno alla Madalena, che lì rimaneva di terra, che andasse avanti. E visto La Crestina, che era investita dalla $\mathrm{d}(\mathrm{ett}) \mathrm{a}$ Galera Turchesca, levò remi sì come feceno tutte le Galere, dandoli buona salma di Moschetate, difendendosi con Mirabil 
valore et intrepidissimo ardire; scorrendo il s(ign)o(r) Cavalire Alesandro Lodi, Cap(ita)no di essa, p(er) tutto con la spada nuda, facendo rinfrescare La Moschettaria senza mai restare.

[32] Finalm(en)te i Turchi ritirandosi, la Magior parte Morti et il resto maltrattati, impauriti con havere rice[v]uto gran danno dalla n(ost)ra Capitana, che di mira et a Man salda uccideva e feriva i Turchi dalla Poppa, sì come faceva La Crestina, che feceno siare, no(n) havendo più ardire tornare allo abordo. Intanto il s(ign)o(r) Generale, tempestando tutta via con le Moschetate, La Crestina, La Capit(an)a, La Padrona, Madalena con altre Galere, datoli il Cavo p(er) Poppa, fece Vela facendo Strada con la Forzza et con l'Armi al dispetto de' Turchi. La ridusse in salvo, passando sotto le prue delle Loro Galere, che con Cannonate e Moschetate continouorno la Battag(li)a. Respondendolit dalle nostre Galere nella stessa Maniera, si uscì allo Mare, no(n) havendo ha[v]uto ardire alcune di esse venire ad investire e di acostarsi più di Mezzo tiro di Moschetto. E preso Caccia, lasceando senza far molta forza le Galere come scoglio, in 3 hore si perseno quasi di Vista, essendo il Vento fresco a Mare grosso. Onde Loro, amainando, Lasorno il seguitarci e, fatto Carro, tornorno a Porto Quaglio, essendoci prima ad una delle Loro Galere rotto l'Albero della Maestra et l'Antenna in tronco del Trinchetto. Il che visto dalle n(ost)re genti che era(no) sop(r)a li ${ }^{u}$ Vascelli, no(n) potendo a tanta forza resistere, si salvorno in terra, et i Turchi, ripresi i Vascelli abandonati, ne diventeno ${ }^{v}$ Patroni e quivi diedeno fondo.

[33] Ma vedendo il s(ign)o(r) Generale no(n) esser più seguitato, reso il Bordo in terra di proprio consiglio, et andò a vista de' nemici 10 Miglia Lontano, a dar fondo $\mathrm{p}(\mathrm{er})$ recuperare le genti lassate, che furno da' Mainotti ${ }^{w}$ e particolarm(en)te da Monsignore Christofano, Arcivescovo di tutto il Cavo del rito Greco, condotti in salvo $\mathrm{p}(\mathrm{er})$ quelle Montagne e Menati alle Galere con esempio di Fede, Carità, e Vera Pietà Christiana, essendo Loro in mezzo a paese di Turchi e loro Vassalli. E recuperati tutti senza perdere alcuno de' nostri, si diede fondo alla punta ove, Mentre si dimorava, si vidde 4 Galere su le 17 hore uscir fuori e far Vela con il rimburcio di 2 delli detti Vascelli, e su le 20 hore uscire le altre 11 con gli altri 4 Vascelli, e retornorno dove erano venuti. E Mentre si stava sul ferro, vennero qui Mainotti alle Galere e fra essi uno che era stato sop(r)a le Galere Turchesche, dove haveva uno suo parente stiavo, e disse come della Galera che investì s(ant)a Crestina n'era Morti nella fattione 40 Turchi, con u(n) Comito et u(n) Rais, et che quasi erano tutti feriti e le Curme maltrattate e n'era di esse Morte Molte. E che tutto haveva inteso dalli stiavi Cristiani. E delle altre turchesche n'era ferite assai, e qualcheduno Morto era, che no(n) sapeva la quantità. Della s(ant)a Crestina ne morseno allora dui soldati e 3 Legati, 9 Feriti di Curma e 9 di scapoli.

[34] Questa Galera era venuta a posta di Costantinopuli, et erano tutte di fanale comandate dal figliolo del Bei di Rodi, venute alla Coda in Barca nostra.

[35] Et havendo il Sig(nor) Generale reordinato le Cose, sul fare della notte

${ }^{t} M S$ respondendoli written over respondendosi

"MS li written over 11

${ }^{v} M S$ diventeno written over divendeno

${ }^{w} \mathrm{MS}$ Mainottti, with 3 t's, written over Mainoti 
sarpò et andò a dar fondo a Vitulo. E la Matt(in)a de 17, da' Mainotti ${ }^{x}$ del paese si intese conforme le nuove di Aptì Bascià sotto Modone. E, levandosi la sera, si fece Vela verso la sapienza che si afferrò la Matt(in)a de 18. E poco doppo venne $\mathrm{u}(\mathrm{n})$ Caecho di Greci di Modone mandati a posta da Aptì Bascià a riconoscere che Galere erano le nostre. Affermorno che erano 20 giorni che quivi si trovava, venuto di Barbaria p(er) passare a Costantinopuli; a' quali il s(ign)o(r) Generale con ogni amorevolezza rimandolli.

[36] E venuto la sera, si sarpò verso le stanfane, che a 3 hore di sole il dì 19 si surse. E rinfrescato l'Acqua, sentì nuova da' Monaci di quel monastero che nel Golfo di Venetia erano state vedute 12 Galere di Barbaria; p(er) la qual cosa il s(ign)o(r) Generale spedì la Filuca al Zante, a peglar lengua; che tornò la matt(in)a de 20 , referì havere inteso che la nuova era come in Barbaria armavano 12 Vascelli, ma no(n) già s'erano veduti in que' Mari, ma sì bene che $\mathrm{p}(\mathrm{er})$ tale effetto s'erano spalmate 10 Galere e 2 Galeazze di Venetia a Corfù, e che quivi aspectavano altre Galere di quella Replubica, e che dovevano condurre in quel'Isola il nuovo proved(dito)re.

[37] E su le 22 hore si sarpò verso il Zante, e vi si diede fondo la Matt(in)a de 21 alla spiaggia, \& vi si intese che di Calabria tenevano nuove che 6 Galere di Biserta et $\mathrm{u}(\mathrm{n}) \mathrm{Brig}($ antino) havevano messo in terra vicino a Napuli, senza nessuno acquisto, con qualche perdita Loro di gente; sì come havevano il Medesimo fatto ne l'Isola di secilia con la medesima sorte. Di già come una Galeotta della previsa di 20 Banchi era venuta a s(ant)a Maura p(er) fornire l'armam(en)to.

[38] E fatto vela su la sera, si andò alla ciaffalonia e vi si dette fondo la Matt(in)a de 22. ${ }^{z}$ Si spedì la Filuca alla Ciaffalonia peccola $\mathrm{p}(\mathrm{er})$ intendere qualche nuova, la quale tornò e referì le stesse nuove che sop(r)a. E p(er) esser il tempo cattivo, si surse a porto Viscardo et, abonaciato $\mathrm{u}(\mathrm{n})$ poco, si sarpò a Mezzanotte verso C(apo) S(ant)a Maria, che essendosi allargato da 30 miglia, mettendosi il Vento fresco di ponente, fu necessario di rendere il Bordo ver[s]o del C(apo) ducato, ove si giunse la Matt(in)a de 23 .

[39] Né di quivi si potette partire fino a 27 all'alba, andando verso Corfù, dove si de' fondo a C(apo) Biancho la sera p(er) disfacere a l'Isola. E preso u(n) poco di reposo, alla 2.a di notte si sarpò tirando verso Italia, che, paregiato il Fano sull'alba de 28, seguendo a Camino la stessa sera si giunse a C(apo) S(ant)a Maria. E da' paesani si intesero confirmare leaa stesse nuove delle Galere Bicertine.

[40] E dimorati in quel Luogo c(irc)a 2 hore, si fece di nuovo Vela, e la Matt(in)a de 29 su le 15 hore si paregiò Gallipoli. E tornando a Camino, cir[c]a a cinq(ue) hore ${ }^{b b}$ di notte, la medesima sera si surse alle Isole di Taranto, ove dato fondo. La matt(in)a seguente ${ }^{c c}$ de 30 , si sarpò e s'andò a salutare la Città e fortezza, e si prese la pratica senza difficultà \&c.

${ }^{x} M S$ daMainotti written over dalMainotti

${ }^{y} \mathrm{MS}$ sula

${ }^{z} M S$ alla, crossed out

${ }^{a} M S$ le written over li

${ }^{b b} \mathrm{MS}$ hore written over hora

${ }^{c} M S$ seguente written over seguenti 


\section{TRANSLATION}

A Journey to the Levant of the 6 Galleys of the Most Illustrious and Holy Order of Santo Stefano, Which Belong to the Most Serene Grand Duke of Tuscany, in This Year 1627, under the Command of the Most Illustrious Lord Giulio Barbolani de' Conti di Montauto, Gentleman, etc.

[1] After having been properly payed and prepared for sailing on April 12th in Leghorn, and having been provisioned with everything that was necessary for them to accomplish their mission, the 6 galleys, which were destined to engage in privateering against the common enemy, could not leave until April 19th because of foul weather. This they did, accompanied by the galley of Santa Maria of the Supreme and Sacred Order of Malta and the new galley San Carlo, which had been given to the above-mentioned order by the Most Serene Prince Don Giovanni Carlo Medici, the grand prior of Pisa.

[2] That same night, at 3:00 a.m., the galleys reached Portoferraio, and here the new lord prince of Piombino and the pursers disembarked. However, because of an adverse wind we were not able to leave until 10:00 o'clock on the morning of the 20th, setting sail toward Monte Argentario.

[3] As we neared the Roman shores, we were forced by inclement weather to enter the harbor of Civitavecchia on the morning of the $21 \mathrm{st}$. We were unable to leave that city until the 26th at dawn, and, once we had passed the Roman coast without incident, we again encountered adverse wind and sea for which it was necessary to anchor in Gaeta, and there we remained until the morning of the 28 th, when we set sail.

[4] When we arrived at Nisita, ${ }^{1}$ the galleys from Malta left us; after having bid farewell to the flagship, they proceeded toward Naples, and we, continuing our journey, went to Capri, where, encountering a rough sea and an adverse wind, we dropped anchor. But the weather got so bad that on the 30 th we turned back and dropped anchor at Mar Morto, ${ }^{2}$ from which we were not able to leave until May 4 th before daybreak.

[5] Crossing the Gulf of Salerno, we entered Messina on the morning of the 5 th, and toward evening of the following day we set sail for the Levant. On the 7 th, at sunrise, we encountered a French polacre, which had left Smyrna twelve days earlier and was going to Marseilles. They reported that the Bey of Rhodes had gone to Constantinople with 14 galleys, that the convoy of Alexandria had not yet left, and that in all of the Levant health conditions were good.

[6] Having left them to their journey, we continued on our route. In the Gulf of Squillace we sighted 3 square-sail vessels, but because of the brisk wind, rough seas, and the late hour we were unable to identify them. That evening we dropped anchor at Cavo Colonna, ${ }^{3}$ and on the morning of the 8 th, we saw again 3 vessels, the same ones we had seen the previous evening. As we set sail to go identify them,

\footnotetext{
${ }^{\mathrm{I}}$ Modern Nisida, an isthmus northwest of Naples off Capo Posillipo in the Gulf of Pozzuoli opposite Capo Miseno.

${ }^{2}$ This is now known as Lago Miseno near Baia on the promontory northwest of Naples.

${ }^{3}$ Capo Colonne is located on a promontory southeast of Crotone in Calabria.
} 
we were forced to return to the Cavo because of bad weather, and there we remained until dawn on the 9th, when we set sail, and that same evening we arrived in Taranto. The order was given to pay and properly prepare the galleys, but this task was postponed until the 12 th because of bad weather. Three galleys were payed that day, and on the 13 th the other 3 were similarly readied.

[7] Having replenished the hold with hardtack, wines, meat, and other edibles, we set sail at 1:00 a.m. in the direction of Capo Santa Maria. ${ }^{4}$ However, because of rough seas and a very brisk and adverse wind, we had to drop anchor at Gallipoli on the 15th. Remaining here for the rest of that day and part of the night, we set sail and dropped anchor upon our arrival at Capo Santa Maria on the 16th. However, during the night a sudden storm arose with rough seas and a foul wind, and in order to escape danger, we had to set sail at midnight, reach the open sea, and return to Gallipoli, where we arrived on the morning of the $17 \mathrm{th}$. We dropped anchor at the island, ${ }^{5}$ where the storm concentrated its force, continuing to rage on both at the island and in the city.

[8] With the return of calm weather, we set sail on the 20th, and on the morning of the 21 st we dropped anchor at the above mentioned Capo, and around 5:00 p.m. the landing party returned and brought a man from the town to the flagship. This man told the admiral that he had been sent by the governor of Otranto that very morning to recommend that the coastal communities keep good watch, since that same day 13 galleys had been sighted at sea. These ships were coming from the gulf toward the Cavo and were believed to be from the Barbary States. The admiral warned all our galleys about this; however, we generally felt that this news was of little importance, and even if the sighting was reliable, it was very likely that they were Venetian galleys.

[9] We stayed anchored there until 3:00 a.m., when a square-sail vessel was sighted just outside the Cavo, and we pursued this vessel. And the crew on this ship, having sighted our galleys, readied their life boat and abandoned ship; everyone reached safety on land, but later — assured of their safety - they returned on board their vessel, a Venetian ship, which had left Manfredonia bound for Naples four days earlier, loaded with grain. They gave us no news.

[10] We left it to its own course, and since the weather was good, with the agreement of all the galleys, we prepared to leave. Crossing the gulf, which was a little rough because of the sea at night, we reached Cephalonia at dawn on the 22nd; and, after resting for a while, we arrived at Zante that same night at 11:00 o'clock. We dropped anchor near the beach and sent the felucca to bring back news from the town. Upon its return, the crew of the felucca told the story of how two months earlier, within the space of thirteen days, two Turkish galleys had been commandeered by mutinous Christian slaves, and both ships-because of stormy weather-had gone to Candia. ${ }^{6}$ On the galley belonging to the Bey of Kavalla ${ }^{7}$ all the Christians were safe, and two of these survivors were taken aboard our galleys

\footnotetext{
${ }^{4}$ Santa Maria is located on the heel of the Italian boot. This site is known today as Santa Maria di Leuca.

${ }^{5} \mathrm{~S}$. Andrea, a small island west of Gallipoli.

${ }^{6}$ Island of Crete.

${ }^{7}$ Seaport in the northeast section of Greece.
} 
by the admiral in order to bring them back home to Leghorn. The other galley, which came from Negropont, ${ }^{8}$ had been given back to the Turks.

[11] Having left at night, we anchored at the Stanfane ${ }^{9}$ on the morning of the 23rd. The Holy Mass was celebrated, and a new supply of fresh water was loaded. After our meal we set sail for Capo di Maina, ${ }^{10}$ where we anchored on the 24 th. We learned from the people of Maina that at Corrone ${ }^{11}$ there were a 24-bench Turkish galiot and a 16-bench brigantine. While we were at anchor there, two other people from Maina came and reported that 3 saics and one caramoussal were at Capo Sant'Angelo, ${ }^{12}$ and they believed that these were Turkish ships which had anchored in that spot.

[12] For this reason the admiral sent the felucca to identify the ships. It returned and confirmed that they were anchored there. Thus, the admiral ordered that 4 caiques be readied and sent together with the felucca to that place, in order that no Turks who were on land might escape, and he sailed after them with our galleys.

[13] However, at dawn some small boats from this group of ships came to our galleys and reported that they were Greek vessels from Saint John of Patmos and from Metellino. ${ }^{13}$ They had no Turks on board nor any Turkish goods, as they were able to prove; they were carrying spun cotton and nut galls, ${ }^{14}$ which they had loaded at Leros ${ }^{15}$ to take to Ancona and Venice, and they had left 12 days earlier. They also told us that in the archipelago at that moment were only 7 galleys, under the command of the Bey of Rhodes, for all the others had sailed to the Black Sea together with approximately 4 small vessels, because of the recent mobilization of troops in that area by the Russians. ${ }^{16}$ They also reported that the above-mentioned Bey of Rhodes had escorted a galleon loaded with gunpowder, cannon balls, and other war matériel almost all the way to Alexandria; from there it was to go to the Turkish encampment in Asia, where we heard that not only Persians but also many rebels were fighting the Turks. Finally, they reported that in the whole area a good state of health was prevailing.

[14] Having left them to their business, we dropped anchor here, but shortly afterwards the coast guard signaled the approach of lateen-rigged vessels. Having arrived at the flagship, they explained that near the Isola dei Cervi ${ }^{17}$ four lateenrigged vessels had been sighted, and these, from their size and their wide wake, were thought to be galleys. For this reason, we set sail in that direction. Outside of Capo Sant'Angelo we sighted two square-sail vessels which, once we began to pursue them, we recognized as the 2 caramoussals of Saint John of Patmos which

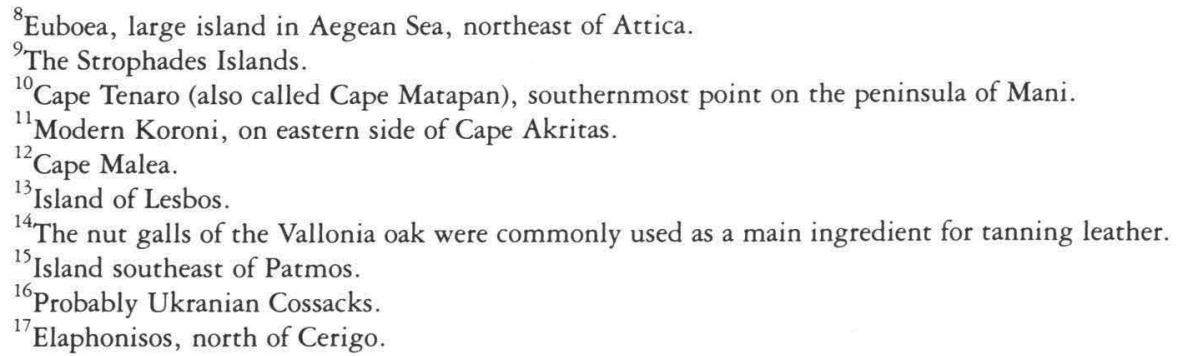


were coming from $\mathrm{Zia} ;{ }^{18}$ they had left five days earlier and were carrying nut galls to Venice. They reported that around noon they had sighted in the channel between Cerigo and the Isola dei Cervi two Venetian galleasses, which were sailing toward Cerigo.

[15] Leaving them to their journey, we continued our route and arrived at Isola Bregiata ${ }^{19}$ on the morning of the $26 \mathrm{th}$. After the rowers had rested, we lowered our sails and set out toward Capo delle Colonne ${ }^{20}$ around 8:00 p.m. Having anchored there on the morning of the $27 \mathrm{th}$, we found two small boats belonging to Greek fishermen from Malvagia, ${ }^{21}$ who had been at sea for three days. They told us that at Napoli di Romania ${ }^{22} 6$ galleys belonging to as many Beys had been payed and made seaworthy there and that these ships had gone to Constantinople to reach the Black Sea and to fight against the Russians, who with a large number of saics were winning many victories in Turkish domains; and furthermore, that from that same city six days previously, three caramoussals carrying 600 janissaries had left for that same purpose.

[16] Leaving them here, we set sail in the evening for Negropont, and at dawn on the 28th we arrived in Planisto. ${ }^{23}$ Having drawn fresh water from a river, we turned back because of bad weather and dropped anchor at Castri. ${ }^{24}$ On the same Island we found a caique loaded with cheese and oil, belonging to some Turks and some Greeks [ . . . . And the Turks escaped to land. We took that which belonged to the Turks and left the rest. We learned nothing of any importance, except that it had come from Napoli di Romania.

[17] While we were anchored here, around noon a Greek Christian slave arrived alone in a small boat. He had fled from Zaychara ${ }^{25}$ four days before; from him we learned the same news reported above. The admiral had him board the flagship and then had his boat sunk.

[18] While we were here on the 31st, we sighted a lateen-rigged vessel in the channel near Andros. However, the very brisk wind and rough sea did not allow us to go and identify it. Finally, the sea and the wind calmed down, and on June 2nd, we set sail after our meal toward Capo d'Oro; we dropped anchor here to allow the rowers to rest. At one o'clock at night we set sail toward Isola di Gazzela, ${ }^{26}$ which we reached at dawn on the $3 \mathrm{rd}$. While we were anchored here, the coast guard came to give us news of a vessel, against which - because it was small-we sent our felucca with an armed caique. These boats captured it and took it back in tow to the flagship. It belonged to some Greeks from Tasso, and we learned from them

${ }^{18} \mathrm{Kea}$, island east of Cape Sunion.

${ }^{19}$ Island of Falconera, west of Milos.

${ }^{20}$ Cape Sunion.

${ }^{21}$ Modern Monemvasia.

${ }^{22}$ Nafplio.

${ }^{23}$ Town on Euboea, known today as Platanistos (?).

${ }^{24} \mathrm{Small}$ island, west of Karistos promontory.

${ }^{25}$ We have been unsuccessful in identifying Zaychara. It could possibly be Zagara near Mt. Elikònas, or the town of Zajeĉar in Yugoslavia.

${ }^{26}$ We have been unsuccessful in our attempts to identify this island. However, it must lie between Negropont and Iura de' Diavoli. 
that they had set sail ten days before and that they were going to Scopullo ${ }^{27}$ to sell pitch. They confirmed the same news reported above.

[19] We left them here, ordering them not to leave, and that same evening we set sail toward Iura de' Diavoli, ${ }^{28}$ where we arrived around midnight. The felucca went ahead to identify the inlet and sighted an armed caique on that same island. The caique, having sighted our galleys, then rapidly went toward the rocky shore, where it ran aground. In their haste, three Turks threw themselves into the sea and were taken aboard the felucca and brought to the flagship. Another 13 Turks found safety on land, and among them was the Aga of Santa Maura, ${ }^{29}$ who-because he was old and lame - could not flee. However, since the felucca wanted to return and board their vessel, the above-mentioned Aga took a musket loaded with small and large shot and fired it at our people. He wounded as many of our men as there were in the felucca, and almost all mortally. One of these, who died within a few days, was the captain of the felucca. The other 13 Turks climbed the steep slopes up through those harsh rocks and with the aid of darkness found refuge in the mountains. As soon as daylight came, we sent soldiers and sailors on land. Within a few hours they brought back to the galleys 6 more Turks, but were unable to find the others. And since night was rapidly approaching, everyone was called back to the galleys, though one of our archers ${ }^{30}$ remained on land, and it was thought either that he had been killed by fleeing Turks, or that he had broken his neck falling from some cliff.

[20] Having requested information from them, we learned that their caique was coming from Gallipoli ${ }^{31}$ and had left the morning before. On board there were 17 Turkish passengers, among whom was the above-mentioned Aga, and the deputy $^{32}$ of the admiral ${ }^{33}$ of the fleet, who was not found. We also learned that 10 galleys with 40 frigates had gone to the Black Sea fifteen days earlier, and that the lord supreme commander was about to leave with another 50 galleys for the same purpose, and it was expected that he would leave for that same place on Friday June 4th. We also learned that Alì Bascià with 150,000 Turks had moved against the Persian, and that in the entire region a good state of health prevailed. After having plundered the vessel, we sighted two other smaller Greek vessels carrying grain, which had been loaded in Cassandra, and they were going toward Napoli [di Romania] and Malvagia. When night came, we left for Tenido, setting sail in the early morning hours of the 5th. At daybreak we saw two saics in the channel between Lendo ${ }^{34}$ and Santo Strati, ${ }^{35}$ and pursuing them and overtaking them, we identified them as Greeks from Schiatta, ${ }^{36}$ who were arriving that morning from Santo Strati, and who had left Constantinople five days earlier. They said that on

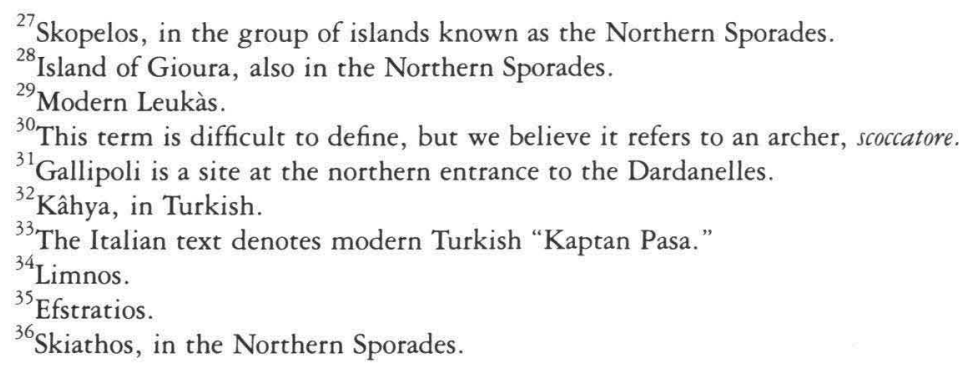


the previous morning they had sighted a galiot at the above-mentioned island. After having taken on fresh water, the galiot entered the channel, at the mouth of which there were 34 vessels both Turkish and Christian. Part of them belonged to the convoy of Alexandria, and among these 2 galleons and four "urche" 37 were unable to enter the channel because of brisk northeasterly winds. They confirmed the same news about the Turkish fleet and about the army going to fight the Persian, as mentioned above.[ . . . ]

[21] Some monks related on their sworn words how in the church of Santa Sophia, now desecrated as a mosque by the Turks, an image painted on wood of the Glorious Mother the Virgin Mary had been miraculously found. When this was seen by the Turks, they threw it into the sea. However, the image returned to its original location through a wondrous miracle. This was seen by the Turks, and when they were deliberating whether to have it removed again, a squad leader ${ }^{38}$ who was in the group asked a janissary what that image was. Coming forward through divine will, the janissary explained that that was the image of Virgin Mary, Mother of Christ, God of the Christians. Whereupon the above-mentioned squad leader, calling him "Dog!" pulled out his scimitar and killed the janissary. Having sliced the image up in many pieces, he had it and the dead man tossed into the sea. However, the next morning the Turks, having again entered the mosque to perform their false ceremonies, saw the janissary kneeling before the whole, restored image, which had returned to its previous position in its original, perfect form, which now had, in addition, a cross on top. Immediately, the order to close off the church was given by the Grand Signior, and harsh punishment would be imposed on anyone, Turkish or Christian, who would try to approach the church or to go inside, in order to give food or drink through a narrow opening to the janissary, who, all alone, was serving there with a golden lamp, which had been made by the Grand Signior and which burned continuously. I wanted to describe this event in such detail, in order that one may with greater clarity comprehend the pure and simple truth of the real miracle which did occur. And of this miracle, as of other great wonders, there is much talk in Constantinople.

[22] Having left them to their journey, we continued our route, sailing the entire day and all of the following night. Having traveled the entire day with our sails furled, we arrived at [Capo] Giannizzero ${ }^{39}$ on the morning of the 6th, and there we sighted the already-mentioned vessels, 22 of which were "urche," caramoussals, "germe" 40 and saics belonging to the Turks. When they sighted our galleys, the Turks abandoned their ships and sought safety on land.

[23] We left the Patrona, Santo Francesco, and Santa Maria Maddalena with the larger part of the Turkish fleet, which was at the entrance [to the channel]. With the other 3 galleys we entered, pursuing the other ships that were seeking safety; but these, too, were abandoned. They were an "urca" with 7 caramoussals, and these vessels, after we had taken the best items from them, were towed

\footnotetext{
${ }^{37}$ Sailing ships of Flemish or Dutch origin which were not particularly known for their nautical abilities (cf. "hulk" in English).

${ }^{38}$ Italian "chiausse," modern Turkish "chavush."

${ }^{39}$ Promontory at the entrance to the Dardanelles, site of ancient Sigeum.

${ }^{40} \mathrm{~A}$ very wide, levantine merchant ship with four sails (Arabic $\hat{g} a r m$ ).
} 
together with the "urca" out [of the channel], and we dropped anchor at the above mentioned Cavo. After we removed the best items-which were, however, very few-from the caramoussals and the saics, the admiral commandeered 4 "urche," a large "germa" and a polacre, all of which were loaded with expensive goods and precious objects. We took only seven prisoners and abandoned the others to their fate at sea, for their ships carried nothing except quantities of rice and similar things.

[24] On the next day, we set sail toward evening, each vessel towing one of the captured ships. This was truly impressive booty; indeed, in human memory booty of this magnitude has never been taken by the entire fleet, and, from what we were able to learn from our prisoners, the value surpassed 700,000 scudi.

[25] Continuing our journey, on the evening of the 9th bad weather forced us to take harbor at San Giorgio on the island of Skyros. On the morning of the 10th, since our supply of fresh water was very low, we went to another inlet, which was not far away, and there replenished our supply. We had left the vessels in tow at the harbor; while returning there, we discovered two saics at sea, which were about to land on shore. As we neared them, we identified them as Greek ships from Molivo on the island of Mitellino, loaded with nut galls for Venice. They told us that when they were in that place on the previous Sunday, an armed caique arrived, which had been sent with great haste by the Aga of Tenido, to warn the rest of the convoy which was behind-that is to say, one galleon with other vessels and 3 galleys, carrying great treasure - that the above-mentioned galleon should take care to keep the above-mentioned galleys in tow. The other vessels had anchored there, claiming that they had heard in that place the sound of cannons. They gave us no other news, and they, too, dropped anchor.

[26] However, when favorable weather returned around midnight, we left harbor and set sail toward Capo d'Oro, and, once we cleared it, we continued our journey. Having passed Isola Bregiata, at midday on the 13 th it was necessary to drop anchor at Capo Sant'Angelo, where we encountered an Albanian who told us that 10 days ago Apti ${ }^{41}$ Bascià had come with his galleys from the Barbary States. And once he had reached Modone, because he learned that we were in the general area, he stopped. In the evening a caique came here from Cerigo with some local people and reported that seven days earlier Vinagro ${ }^{42}$ together with a galley had passed by, going toward the Barbary States. We set sail around midnight heading toward Isola dei Cervi, and because of foul weather we had to anchor there.

[27] While there, we discovered in the waters near Cerigo a square-sail vessel; when our galleys went out to identify it, it turned out to be an English vessel which, coming from Leghorn, had been at sea for fourteen days and was going to Smyrna; it had formed a convoy with the vessel of the 11 knights of Brother Gualterotto Bardi and of Brother Nicola Gianfigliazzi as far as Navarino, ${ }^{43}$ and they had parted company three days before.

[28] Having left them to their course, we returned to the harbor. We left at

\footnotetext{
${ }^{41}$ Corresponding to the modern Turkish proper name Abdi.

${ }^{42}$ The name by which the infamous Turkish warship, the "Capitana di Scio," was known (cf. Guarnieri 203).

${ }^{43}$ Modern Pylos.
} 
night, setting sail toward Capo di Maina, where we arrived in the morning of the 15 th. And remaining there all day and part of the night and wishing to leave, we were unable to do so because of foul weather and also because one of the vessels had become entangled with the anchor of the flagship, and they were in that predicament for about seven hours.

[29] However, though we were preparing to leave at dawn on the morning of the 16th we sighted near the eastern promontory a galley that was coming toward the harbor. Immediately, the admiral ordered the Patrona, the Santo Francesco, and the Santo Stefano to set sail, since the anchor rope of the flagship had once again become entangled and it was necessary to cut the rope off and abandon the anchor.

[30] Once the Maria Maddalena and the Cristina had set sail, the flagship left. However, the galley Santo Francesco, being further ahead than the others, sighted other galleys and fired an appropriate warning shot, just as the above-mentioned galley did to the rest of the convoy. Suddenly around the promontory there began to come into view another 14 galleys displaying the "fanale," toward us in full force to break up our formation and ram us, firing many cannon shots. We escaped without damage, thanks to God.

[31] However, although the enemy galley pursued our flagship, which gave it a fierce battle with frequent volleys of musket shots, the enemy vessel, contrary to its expectations, could not catch up with our flagship. Having suffered damage and fearing to receive even more, the enemy galley lifted its oars and set sail. As our flagship had gained the advantage, the above-mentioned galley turned toward the Cristina. Seeing the closeness of this pursuit, the admiral signaled to the Maddalena, which was closer to shore, to move forward. And the Cristina, seeing that it had been attacked by the above-mentioned Turkish galley, raised its oars out of the water, just as all of our other galleys did, and fired a good volley of musketshots, defending itself with remarkable courage and very intrepid bravery. Signor Cavaliere Alessandro Lodi, the captain [of the Cristina, ] was chasing about with his drawn sword and giving orders that the musketeers continue firing without pause. [32] At last the Turks retreated, most of them dead and the remainder in a pitiful state and scared because of the great damage suffered from our flagship, which, through gunfire and hand-to-hand combat, was killing and wounding the Turks from the stern, just as the Cristina was doing. No longer having the courage to renew their attack, the Turks rowed away in retreat. Meanwhile the admiral ordered continued volleys of musket shots to be fired at them, and the Cristina, the flagship, the Patrona, and the Maddalena, together with the other galleys, having set their course away from the Cavo, set sail and made their way with force and show of arms in defiance of the Turks. The admiral was able to conduct the ships to safety passing under the prows of the enemy galleys which continued the battle with volleys from cannons and muskets. Making a similar response from our galleys, we succeeded in reaching open sea, since none of their ships had been bold enough to come ram us or to come closer than half a musket shot. Having undertaken to escape pursuit, without a great deal of effort we left their galleys

\footnotetext{
${ }^{44}$ The "fanale" is a special kind of light or lantern which indicated the presence on board of high ranking officials.
} 
behind us as though they were rocks, and in 3 hours they were almost out of sight, for the breeze was brisk and the sea rough. For this reason they furled their sails and stopped following us, and having made the proper maneuver with the mast and boom, they returned to Porto Quaglio, since earlier one of their galleys had sustained a broken mainmast and yard arm on the foremast. This event having been seen by our crew members who were aboard the vessels [in the harbor], and not being able to resist their superior strength, our crew sought safety on shore, and the Turks, having recovered their captured vessels, which we had abandoned, became their owners once again and here they anchored.

[33] However, the admiral, seeing that we were no longer being pursued, turned the ship toward land with good judgment, and went within sight of the enemy-10 miles away-to drop anchor in order to recover the crew members who . had been left behind. They had been led to safety by people from Maina and especially by Monsignor Christofano, archbishop of all the area of the Cavo which followed the Greek rite. The crew had been led to safety through those mountains and brought to the galleys as an example of faith, love and true Christian piety, since all of them were in a country amid Turks and their subjects. And having recovered all of our men without losing anyone, we anchored at the point. While we were there, around 5:00 p.m. we saw 4 galleys, leaving and setting sail with two of the above-mentioned vessels in tow, and, around 8:00 p.m., we saw the remaining 11 galleys leave with the other 4 vessels, and they returned whence they had come. And while we were anchored there, some people from Maina came to our galleys, and among them a man who had been aboard the Turkish galleys, where one of his relatives was a slave. And he told us that on the galley which had engaged the Santa Cristina in battle some 40 Turks had been killed in the encounter, together with a petty officer and a Rais. ${ }^{45}$ Moreover, he said that almost all of them had been wounded and the rowers left in a pitiful state, with many dead among them. He had heard all this from Christian slaves. Furthermore, on the other Turkish vessels there were many wounded and some dead, but he did not know exactly how many. On the Santa Cristina two soldiers and three legates died, nine rowers and nine among the unchained rowers ${ }^{46}$ were wounded.

[34] This galley [on which his relative served] had come precisely for this purpose from Constantinople, and all these vessels displayed the "fanale" and were under the command of the young son of the Bey of Rhodes and had been following our ship.

[35] The admiral set things in order, and, as night approached, we set sail and went to Vitulo, where we dropped anchor. On the morning of the 17 th we received confirmation from the local people of Maina of the news about Aptì Bascià, sighted near Modone. As night approached, we set sail toward Sapienza, where we anchored on the morning of the 18th. And shortly thereafter a caique with Greeks from Modone arrived which had been sent to the harbor by Aptì Bascià to identify our galleys. They affirmed that they had been here 20 days, having come from the Barbary States on their way to Constantinople. The admiral sent them back to their vessels with great courtesy.

\footnotetext{
${ }^{45}$ Captain (Rais, Arabic).

${ }^{46}$ The term scapoli indicates those rowers who were not slaves and who were paid a daily wage.
} 
[36] And once evening had arrived, we sailed toward the Stanfane, where we landed at 3 hours after sunrise on the 19th. And having taken on a supply of fresh water, we heard news from monks of that monastery that in the Gulf of Venice 12 galleys coming from the Barbary States had been seen; for this reason the admiral sent the felucca to Zante to get new information. It returned on the morning of the 20th and reported that it had learned that the news was that in the Barbary States 12 vessels were being prepared and armed, but that they had not yet been sighted in these seas. Nevertheless, for such an enterprise 10 Venetian galleys and 2 Venetian galleasses had been properly payed and prepared for sailing at Corfù, and there [at Corfù] they were waiting for other galleys from that republic, which were supposed to bring the new governor to that island.

[37] Around 10:00 p.m., we set sail for Zante and anchored there along the beach on the morning of the 21st. There we learned that the news from Calabria was that 6 galleys from Biserta and a brigantine had put ashore near Naples, without any profit and with some loss of men, just as the same vessels had done on the island of Sicily with the same outcome. We also learned how a 20-bench galiot had come to Santa Maura from Previsa to supply armaments.

[38] Having set sail as evening approached, we went to Cephalonia, where we anchored on the morning of the 22 nd. We sent the felucca to Little Cephalonia ${ }^{47}$ to learn some news, and it returned and informed us of the same news reported above. Since the weather was bad, we dropped anchor at Porto Viscardo, and when it had calmed down a bit, we set sail around midnight toward Capo Santa Maria. When we were 30 miles off shore, a brisk west wind came up, and we had to turn our ship toward Capo Ducato, which we reached on the morning of the 23rd.

[39] We were unable to leave from there until dawn on the 27 th, when we headed for Corfù, where we dropped anchor at Capo Bianco in the evening, in order to furl the sails at the island. Having rested a bit, at 2:00 a.m. we set sail for Italy, and having gone by the island of Fano at dawn on the $28 \mathrm{th}$, we continued our route and arrived the same evening at Capo Santa Maria. From the local people we had confirmation of the same news about the galleys from Biserta.

[40] Having remained in that place for about two hours, we again set sail, and during the day of the 29th, around 3:00 p.m., we went by Gallipoli. Continuing on our route for five hours that night, that same evening we came to the Islands of Taranto, where we dropped anchor. The following morning, on the 30th, we set sail and went to give a salute to the city and fortress, and we received all necessary permissions ${ }^{48}$ with no difficulty, etc.

\footnotetext{
${ }^{47}$ Ithaca.

${ }^{48}$ The pratica is the permission to dock and disembark given by health officials.
} 